\title{
An alternative quadratic formula
}

Norbert Hungerbühler

\section{A short history of the quadratic formula}

The commonly used formula to solve a quadratic equation

$$
a x^{2}+b x+c=0, \quad a, b, c \in \mathbb{C}, a \neq 0,
$$

is the quadratic formula

$$
x_{1,2}=\frac{-b \pm \sqrt{b^{2}-4 a c}}{2 a} .
$$

In German speaking countries this formula is called Midnight Formula or Moonshine Formuld* since a student is supposed to know it by heart even when torn from sleep at midnight. From a didactical point of view, this paradigm is certainly disputable since knowing something by heart is the enemy of understanding the subject. This is why Hermann Weyl used to urge his students to "un-learn" this formula.

The formula appeared for the first time in Descartes' Discours de la méthode [4, p. 303] where he coined one of the most enduring conventions of mathematics, namely the use of $a, b, c, \ldots$ as constants and $x, y, z$ as variables (see [2]).

The prehistory of the quadratic formula goes back to Babylonian mathematics: One of the oldest traces is the cuneiform tablet $B M$ 13901, stored in the British Museum, which states around 80 mathematical problems. It dates back to Hammurabi's reign in the beginning of the 18th century BC. The first problem asks to find the side length of a square (see [10] for a detailed discussion):

The surface plus the length of the square is $3 / 4$.

In modern terms this corresponds to the equation $x^{2}+x=3 / 4$. The later Greek mathematics would have never accepted this mixing of dimensions. However, the proposed Babylonian solution may be translated as follows:

Take 1 , the unity. Brake it up in $1 / 2$ and $1 / 2$. Square $1 / 2$ to get $1 / 4$. Add $1 / 4$ to $3 / 4$ and obtain 1 . The root of this is 1 . Subtract of it the $1 / 2$ which you squared before. You get $1 / 2$ which is the side of the square.

These numerical steps are expressed in the formula

$$
\sqrt{(1 / 2)^{2}+3 / 4}-1 / 2=1 / 2
$$

which indeed corresponds to the positive solution of the quadratic equation (see also (5)).

\footnotetext{
*Mitternachtsformel or Mondscheinformel
} 
In Greek mathematics we find geometric methods to solve quadratic equations in Euclid's works: In [5] and [16 the Theorems $5 \& 6$ in Book II and the Theorems $28 \& 29$ in Book VI of the Elements, as well as the Theorems $84 \&$ 85 in the Data are interpreted as tools to solve quadratic equations (see 9 for a critical analysis of this view). However, it is conceivable that Euclid would have rather used the Theorems 35 and 36 in Book III, since they allow a much more direct link between the quadratic equation and its geometric solution: Suppose we want to solve the equation $x^{2}-u x+v w=0$, where $u, v, w>0, u^{2}-4 v w \geq 0$, are given as lengths of segments. Then, the solutions $x_{1}, x_{2}$ satisfy $x_{1}+x_{2}=u$ and $x_{1} x_{2}=v w$. Hence the following application of the intersecting chords theorem yields the solution on the left:

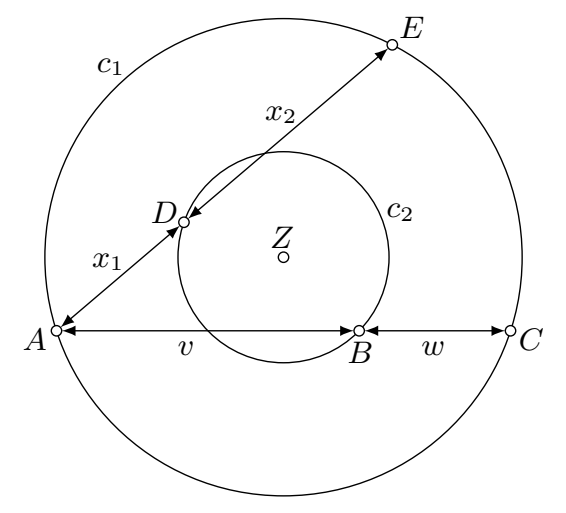

Choose $c_{1}$ to be a sufficiently large circle through $A$ and $C$ and $c_{2}$ a concentric circle through $B$. Then $c_{2}$ cuts a chord $A E$ of length $u$ in segments of lengths $x_{1}, x_{2}$. See also Exercise 4.

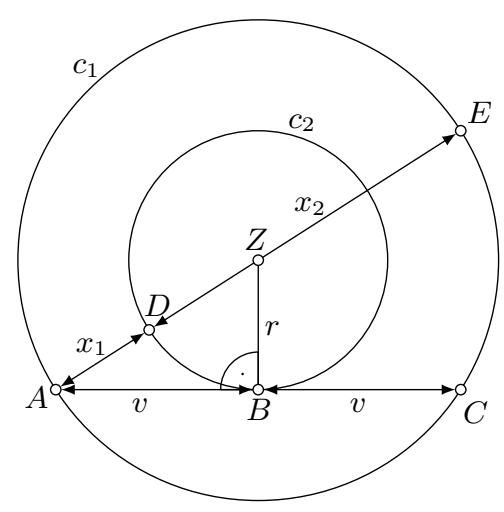

In particular, if we choose $v=w$ and $A E$ as diameter of $c_{1}$, we have $r=\sqrt{(u / 2)^{2}-v^{2}}$ and $|A Z|=u / 2$. Hence we can read off the solutions of $x^{2}-u x+v^{2}=0$ and get the familiar expression $x_{1,2}=u / 2 \pm \sqrt{(u / 2)^{2}-v^{2}}$.

The famous Bakhshali manuscript, the oldest passed down manuscript in Indian mathematics, also contains examples and solutions of quadratic equations with numerical coefficients. This birch bark manuscript is dated between the 9th and the 12th century CE but is assumed to have origins going back to the 3rd or 4th century CE. In Chinese mathematics, the Zhang Qiujian suanjing, a manual from the 5th century CE, not only discusses quadratic equations, it also contains a consistent concept of negative numbers. See 1 for more information.

An important milestone in the history of mathematics is Al-Khwarizmi's algebra book Al-kitāb al-mukhtașar fī hisāb al-ğabr wa'l-muqābala which is, in part, devoted to an extensive discussion and classification of quadratic equations. Unlike in earlier work, Al-Khwarizmi, who lived between 780 and $850 \mathrm{CE}$, introduced the concept of equation not only as a series of case by case problems to be solved, but as an infinite class of problems with a common method of solution. In particular, he identified the cases where the quadratic equation has two, one 
or no solution. He also demonstrated different methods of solution by means of the idea of equal area. It is possible, that he was inspired by the translation of Euclid's Elements by al-Hajjaj ibn Matar who, like Al-Khwarizmi, worked in the House of Wisdom in Baghdad (see [14, Chapter 11]). Although most of the modern notation was not yet available at that time and al-Khwarizmi still formulated his problems and solutions in text form, his work marks the dawn of elementary algebra.

So Descartes was, by far, not the first to solve quadratic equations. However, instead of giving a verbal description of the algorithm which yields the solution from the numerical values of the coefficients of a given single equation, he wrote down the solution as a condensed formula involving the coefficients of the equation as variables. This progress can hardly be overestimated: The formula allows to analyze the solutions as functions of these coefficients, which in turn may depend on other variables. Moreover, one may make use of the formula as an element in more complex computations. Finally, a clear formal justification of the formula becomes possible, not only a case by case verification of the numerical values at hand.

\section{Friends of the quadratic formula}

Quadratic equations can not only be considered over $\mathbb{C}$ but also over a general field. The way to compute the solutions of (2) from the coefficients involves the four basic arithmetic operations and square roots.

Roots. In a general field $F, Q(F):=\left\{x^{2} \mid x \in F\right\}$ is the set of squares. A function $\sqrt{ }{ }^{-}: Q \rightarrow F$ with the property $(\sqrt{x})^{2}=x$ is called a square root. The polynomial $p(x)=x^{2}-r$ has at most two zeros. For $r \in Q(F)$ the two zeros are $\pm \sqrt{r}$, and these two values coincide if $r=0$ or if $F$ has characteristic 2. If $F$ is algebraically closed all elements are squares. By virtue of the Frobenius automorphism the same is true for every finite field of characteristic 2. And in a finite field of odd characteristic there are as many nonzero squares as nonsquares. In that case, products of two squares or of two nonsquares are squares, a product of a square and a nonsquare is a nonsquare.

The axiom of choice, or more precisely the axiom of choice $C_{2}$ for families of pair sets, ensures the existence of a square root for a general field. Actually, $C_{2}$ is equivalent to the fact, that in each field there exists a root on its set of squares (see [7, Proposition 4.3]). However, there are cases where a root may be defined due to additional structures: If $F$ is an ordered field, e.g. $\mathbb{R}$, one may always choose the larger of two possible values for the root of a square. In the case $F=\mathbb{C}$ one can use its structure of a Riemann surface to define a root by identifying an analytical principal branch of the root function.

It is worth noticing that the solution formula (2) works with any choice of a root function and in any field of characteristic $\neq 2$. Let us have a brief look at the special case of a field of characteristic 2 . 
Quadratic equations in a field of characteristic 2. We consider (1) in a field $F$ of characteristic 2. If $b=0$, then $\sqrt{1}$ is equivalent to $x^{2}=c / a$. And if $c / a \in Q(F)$, or equivalently $a c \in Q(F)$, the unique solution in $F$ is $\sqrt{c / a}$. If $a c \notin Q(F)$, there is no solution in $F$.

If $b \neq 0$, we rewrite (1) in the form

$$
\left(\frac{a x}{b}\right)^{2}+\frac{a x}{b}+\frac{a c}{b^{2}}=0
$$

which, for $y:=\frac{a x}{b}$, takes the form

$$
y^{2}+y=\frac{a c}{b^{2}} .
$$

If we consider $F$ as a vector space over its prime field $\mathbb{F}_{2}$, then the Artin-Schreier map $F \rightarrow F, y \mapsto y^{2}+y$, is linear. Its kernel $K$ consists of the elements $z \in F$ for which $z^{2}=z$, i.e., $K=\mathbb{F}_{2}$. Hence, (3) has a solution $y$ iff $a c / b^{2}$ is an element of the image of the Artin-Schreier map, and then $y+1$ is the second solution. In terms of the original variable $x$, we get: (1) with $b \neq 0$ has no solution if $a c / b^{2}$ does not lie in the image of the Artin-Schreier map, and it has the two solutions $\frac{b y}{a}$ and $\frac{b(y+1)}{a}$ if $y$ is a preimage of $a c / b^{2}$ under the Artin-Schreier map. For a detailed discussion, see [3].

Characteristic $\neq \mathbf{2}$. In a general field $F$ of characteristic $\neq 2$, the number of solutions of (1) is governed by the discriminant $D:=b^{2}-4 a c$ : For coefficients $a, b, c \in F$, equation (1) has no solution in $F$ if the discriminant $D$ is a nonsquare. If $D$ is a square, (1) has one solution if $D=0$ otherwise two solutions.

But let us now concentrate to the case $F=\mathbb{C}$ and discuss alternative ways to represent the solutions of (1).

When starting from the normalized form

$$
x^{2}-2 p x+q=0, \quad p, q \in \mathbb{C},
$$

the quadratic formula takes the form

$$
x_{1,2}=p \pm \sqrt{p^{2}-q} .
$$

Lesser-known is the formula

$$
x_{1,2}=\frac{-2 c}{b \pm \sqrt{b^{2}-4 a c}}
$$

for the solutions of (11). This variant follows from (2) by observing, that $x_{1} x_{2}=$ $c / a$. The formula (6) yields only one solution if $c=0$, but otherwise it gives a correct answer even in the degenerate case $a=0$ (i.e. a linear equation), provided $b \neq 0$. For this reason (6) is used in Muller's iterative method [12] for numerically finding roots of a function $f$ by approximating $f$ with parabolas interpolating the graph in three points. 
For real coefficients, trigonometric and hyperbolic methods for solving quadratic equations are also circulating: In contrast to $(2),(5)$ and $(6)$ these formulas are numerically stable (see [13, 6], and the discussion in Section 3). To explain the idea we assume, without loss of generality, that $a>0$ in (1). Moreover, we sort out the cases $b=0$ (with the solutions $x_{1,2}= \pm \sqrt{-c / a}$ ) and $c=0$ (with the solutions $\left.x_{1}=0, x_{2}=-b / a\right)$. Otherwise, we distinguish the cases $c>0$ and $c<0$ :

Case 1. If $c<0$ we bring equation $\sqrt{1}$ by the scaling $x=y \sqrt{-\frac{c}{a}}$ to the normalized form

$$
y^{2}+d y-1=0, \text { with } d=\frac{b}{\sqrt{-a c}} .
$$

Using the double-angle formula

$$
\tan 2 \alpha=\frac{2 y}{1-y^{2}} \quad \text { for } y=\tan \alpha
$$

we see that $y_{1}=\tan \alpha$ is a solution of 77 if $\alpha$ is chosen such that $\tan 2 \alpha=\frac{2 \sqrt{-a c}}{b}$. According to Viëta's Theorem, the product of the solutions of (7) is $y_{1} y_{2}=-1$, i.e., $y_{2}=-\cot \alpha$. Summarizing, we get as solutions of (1)

$$
x_{1}=\sqrt{-\frac{c}{a}} \tan \alpha, \quad x_{2}=-\sqrt{-\frac{c}{a}} \cot \alpha, \quad \text { with } \tan 2 \alpha=\frac{2 \sqrt{-a c}}{b} .
$$

Case 2. If $c>0$ we first have to sort out the case $b^{2}-4 a c=0$ with double root $x_{1}=x_{2}=-\frac{b}{2 a}$. Otherwise, we bring equation (1) by the scaling $\left.x=y \sqrt{\frac{c}{a}}\right\rfloor^{\dagger}$ to the normalized form

$$
y^{2}+d y+1=0, \text { with } d=\frac{b}{\sqrt{a c}} .
$$

Recall the double-angle formula for the hyperbolic tangent

$$
\tanh 2 \alpha=\frac{2 y}{1+y^{2}} \quad \text { for } y=\tanh \alpha .
$$

The range of the function tanh is $\mathbb{C} \backslash\{-1,1\}$. Since $b^{2}-4 a c \neq 0$, we have $\frac{2 \sqrt{a c}}{b} \neq \pm 1$ and we can choose $\alpha$ such that $\tanh 2 \alpha=-\frac{2 \sqrt{a c}}{b}$, and actually $\alpha \in \mathbb{R}$ iff $b^{2}-4 a c>0$. Then, by $(9)$ it follows that $y_{1}=\tanh \alpha$ is a solution of (8). Again by Viëta's Theorem, the product of the solutions of (8) is $y_{1} y_{2}=$ 1, i.e., $y_{2}=\operatorname{coth} \alpha$. Putting all together, we find as solutions of (1) in the considered case

$$
x_{1}=\sqrt{\frac{c}{a}} \tanh \alpha, \quad x_{2}=\sqrt{\frac{c}{a}} \operatorname{coth} \alpha, \quad \text { with } \tanh 2 \alpha=-\frac{2 \sqrt{a c}}{b} .
$$

\footnotetext{
${ }^{\dagger}$ Alternatively, one may use $x=i y \sqrt{\frac{c}{a}}$ to arrive again at the form $y^{2}+d y-1=0$ like in the first case.
} 
It may look strange at first sight to employ trigonometric or hyperbolic functions to solve a quadratic equation. However, recall, that the use of trigonometric functions becomes inevitable for the cubic and quartic equation in the casus irreducibilis. And for the general quintic equation, even trigonometric functions are no longer strong enough: Hermite used the theory of elliptic modular functions and the Jacobi theta function to solve the quintic (see [8]). Later, Klein related the symmetries of the icosahedron with Galois theory to show systematically, why elliptic modular functions appear naturally in the solution of the quintic. He also devised a solution using hypergeometric functions (see [1]).

\section{Meet a new friend}

Here, we want to propose an alternative quadratic formula which stands out by its symmetry.

Proposition. Let the quadratic equation be given by the homogeneous normal form

$$
x^{2}-4 u x+4 v^{2}=0, \quad u, v \in \mathbb{C} .
$$

Then its solutions can be expressed by

$$
x_{1,2}=(\sqrt{u+v} \pm \sqrt{u-v})^{2} \text { or equivalently } x_{1,2}=(\sqrt{u-v} \pm \sqrt{u+v})^{2} .
$$

For real coefficients $u, v^{2} \in \mathbb{R}, 10$ has

(a) a double root (namely $2 u$ ) iff $u^{2}=v^{2}$,

(b) two positive distinct real roots iff $u^{2}>v^{2}$,

(c) two real roots of opposite sign iff $v^{2}<0$.

Remark. It is easy to convert the standard form (1) to the homogeneous form (10), however it requires a root to determine $v$. But for dimensional reasons, problems originating from physics or geometry often present themselves already in the form (10). Actually, Descartes' first example at the very hour of birth of the quadratic formula, was geometric and of the homogeneous form 10 with coefficients $u=\frac{a}{4}, v=\frac{b}{2}$ : The following facsimile shows the corresponding excerpt of page 303 in [4]. 


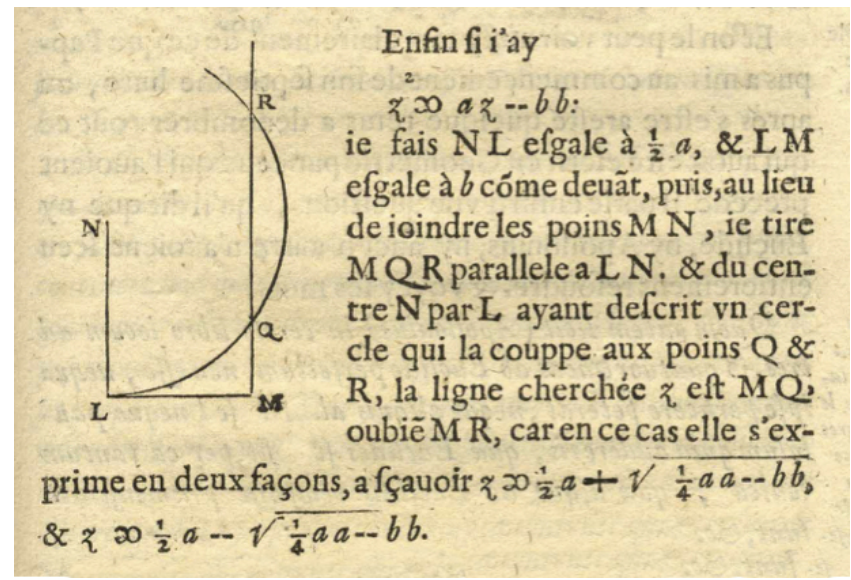

Proof of the Proposition. The classical formula (2) gives initially

$$
x_{1,2}=2 u \pm 2 \sqrt{u^{2}-v^{2}} .
$$

This establishes (a)-(c) in the case of real coefficients $u, v^{2}$. In general, if $u, v^{2} \in$ $\mathbb{C}$, we use that for any root function on $\mathbb{C}$ the product $\sqrt{u+v} \sqrt{u-v}$ is either plus or minus $\sqrt{(u+v)(u-v)}$ and get by 12

$$
\begin{aligned}
x_{1} & =2 u+2 \sqrt{u^{2}-v^{2}}=u+v+u-v+2 \sqrt{(u+v)(u-v)}= \\
& =u+v+u-v \pm 2 \sqrt{u+v} \sqrt{u-v}=(\sqrt{u+v} \pm \sqrt{u-v})^{2}
\end{aligned}
$$

with either the plus or the minus sign. The result for $x_{2}$ is the same expression with the opposite sign.

Alternatively, the correctness of 11 can readily be checked, without transforming the product $\sqrt{u+v} \sqrt{u-v}$, by plugging it into 10 .

A benefit of the new formula (11) compared to the classical version (2) is its numerical stability. To illustrate this property, we compare the classical formula with the new formula when applied to the model problem in [6]

$$
x^{2}-10^{n} x+1=0 .
$$

For simplicity we take $n>1$ even. Then the solution obtained by (2) rounded to $2 n$ significant decimals, are

$$
\begin{array}{ll}
x_{1}=10^{n}-10^{-n} & \text { with a relative error of the order } 10^{-4 n} \\
x_{2}=10^{-n} & \text { with a relative error of the order } 10^{-2 n} .
\end{array}
$$

However, if we repeat the calculation rounded to $2 n-1$ significant decimals, we are victims of catastrophic cancellation and get

$$
\begin{array}{ll}
x_{1}=10^{n} & \text { with a relative error of the order } 10^{-2 n} \\
x_{2}=0 & \text { with a relative error of } 100 \% .
\end{array}
$$


If we use (11) instead, the situation is considerably better, as it still yields satisfactory solutions if the calculations are carried out rounded to only $n$ significant decimals:

$$
\begin{array}{ll}
x_{1}=10^{n} & \text { with a relative error of the order } 10^{-2 n} \\
x_{2}=10^{-n} & \text { with a relative error of the order } 10^{-2 n} .
\end{array}
$$

The following example taken from a physics textbook 15, Section 14.3, Problem 8] shows how nicely (11) may turn out compared to the classical formulas (2) or (5):

Exercise. A stone is dropped from rest into a well. $t$ seconds later, the sound of the splash is heard. Ignoring air resistance, how far has the stone fallen before hitting the water?

Solution. Let $x$ denote the depth of the well, $c$ the velocity of sound, and $g$ the acceleration of fall (we neglect air resistance). The time $t$ is the sum of the time $t_{1}=\sqrt{\frac{2 x}{g}}$ for the stone to fall and the travel time $t_{2}=\frac{x}{c}$ of the sound. Squaring $t_{1}=t-t_{2}$ and rearranging terms leads to the quadratic equation

$$
x^{2}-2 x \frac{c(c+g t)}{g}+t^{2} c^{2}=0 .
$$

The alternative formula (11) yields the particularly compact expression

$$
x_{1}=\frac{c^{2}}{2 g}\left(\sqrt{1+\frac{2 g t}{c}}-1\right)^{2} .
$$

Compare this to the rather messy expression you get from the classical formula (5)! Let us briefly discuss the result: A falling stone reaches after time $\tau=\frac{c}{g}$ the velocity of sound. During that time $\tau$, it travelled the distance $\frac{c^{2}}{2 g}$ which is precisely the first factor in (14). The second factor, the squared bracket, is a dimensionless number. The Taylor expansion in powers of $1 / c$ of 14 is

$$
x_{1}=\frac{g t^{2}}{2}-\frac{g^{2} t^{3}}{2 c}+O\left(1 / c^{2}\right)
$$

Here, the first term is the answer to the exercise in the case of infinite propagation of sound, the second term gives the first order correction (in $1 / c$ ) which yields reasonably good results in practice.

Observe, that the second solution $x_{2}$ does not solve the given physical problem. However, the reader is invited to figure out what physical interpretation the second solution of 13 has.

\section{Exercises}

1. Solve Descartes' problem $z^{2}=a z-b^{2}$ by the alternative formula (11). Interpret the result geometrically. 
2. Let $F$ be a field of characteristic $\neq 2$. Prove: The solutions of the quadratic equation $x^{2}-p x+q=0$ are squares in $F$ if and only if $q=r^{2}$ is a square, and $p \pm 2 r$ are squares.

3. Let $a, b \in \mathbb{Q}$ be positive such that $a^{2}>b, b \notin Q(\mathbb{Q})$. Show that $x, y \in \mathbb{Q}$ with

$$
\sqrt{a \pm \sqrt{b}}=\sqrt{x} \pm \sqrt{y}
$$

exist iff $a^{2}-b \in Q(\mathbb{Q})$. Find $x$ and $y$ in this case.

4. Solve the quadratic equation $x^{2}-u x-v w=0$ with the Intersecting Secants Theorem (Theorem 36 in Book III of Euclid's Elements).

5. A company sells olives in large cardboard boxes folded from squares of sidelength $a$ :

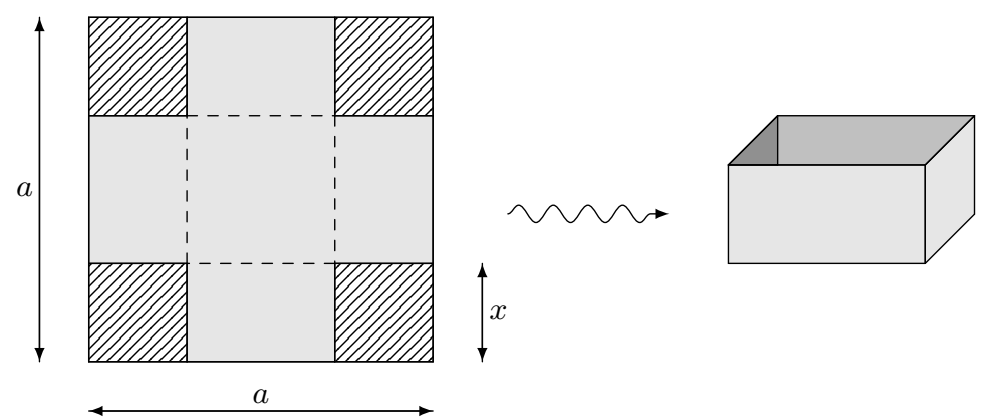

The selling price for olives is $s$ Dollar per unit volume. The price for one box is therefore $p(x)=s x(a-2 x)^{2}$. The cost for the cardboard is $c$ Dollar per unit area. The cost per box is therefore $q(x)=c\left(a^{2}-4 x^{2}\right)$. Optimize the profit $p(x)-q(x)$ per box. Use the alternative formula (11).

Acknowledgement. The author would like to thank Hans Peter Dreyer for pointing out to him the nice exercise of the falling stone, and Jacques Gélinas for the remark about the numerical stability.

\section{References}

[1] Ari Ben-Menahem. Historical Encyclopedia of Natural and Mathematical Sciences, volume 1. Springer, 2009.

[2] Bill Casselman. x, y, and z. Notices Amer. Math. Soc., 2015.

[3] Jørgen Cherly, Luis Gallardo, Leonid Vaserstein, and Ethel Wheland. Solving quadratic equations over polynomial rings of characteristic two. Publ. Mat., 42(1):131-142, 1998. 
[4] Descartes, René. Discours de la Méthode. Imprimerie de Ian Maire, 1637.

[5] Walter C. Eells. Greek Methods of Solving Quadratic Equations. Amer. Math. Monthly, 18(1):3-14, 1911.

[6] George E. Forsythe. Pitfalls in Computation, or why a Math Book isn't Enough. Amer. Math. Monthly, 77(9):931-956, 1970.

[7] Lorenz Halbeisen, Norbert Hungerbühler, Nir Lazarovich, Waltraud Lederle, Marc Lischka, and Salome Schumacher. Forms of choice in ring theory. Results Math., 74 (2019), no. 1, Art. 14, 33 pp.

[8] Charles Hermite. Sulla risoluzione delle equazioni del quinto grado. Annali di math. pura ed appl., 1:256-259, 1858.

[9] Roger Herz-Fischler. What are propositions 84 and 85 of Euclid's data all about? Historia Mathematica, 11:86-91, 1984.

[10] Jens Høyrup. Lengths, widths, surfaces: A portrait of old babylonian algebra and its kin. Springer, 2002.

[11] Felix Klein. Vorlesungen über das Ikosaeder und die Auflösung der Gleichungen vom fünften Grade. Birkhäuser Verlag, Basel; B. G. Teubner, Stuttgart, 1993. Reprint of the 1884 original.

[12] David E. Muller. A method for solving algebraic equations using an automatic computer. Math. Tables Aids Comput., 10:208-215, 1956.

[13] William H. Press, Saul A. Teukolsky, William T. Vetterling, and Brian P. Flannery. Numerical recipes in Fortran 7\%, volume 1 of Fortran Numerical Recipes. Cambridge University Press, Cambridge, second edition, 1992.

[14] R. Rāshid and R. Morelon. Encyclopedia of the History of Arabic Science, volume 2. Routledge, 1996.

[15] Raymond A. Serway, Chris Vuille, and Jerry S. Faughn. College Physics. Brooks/Cole Cengage Learning, 2009.

[16] Bartel L. van der Waerden. Science Awakening I. Noordhoff, 1954.

Norbert Hungerbühler

Department of Mathematics

ETH Zürich

8092 Zürich, Switzerland

norbert.hungerbuehler@math.ethz.ch 